\title{
Physiological Interactions Of Calcium And Vitamin D In Colon
}

Ahmed Saber Abu-Zaiton

Al-albayt University, Jordan

Ahed J Alkhatib

Jordan University of Science and Technology, Jordan

Suha K Ababneh

The University of Jordan, Jordan

Faisal Kanaan

Department of pathology, King Abdulla University Hospital, Jordan

doi: 10.19044/esj.2016.v12n24p49 URL:http://dx.doi.org/10.19044/esj.2016.v12n24p49

\begin{abstract}
This study aimed to review the literature about possible physiological interactions of calcium and vitamin D in colon. Previous studies have indicated to protective effects of vitamin $\mathrm{D}$ and calcium in case of colon cancers. We wanted to check the physiological effects of both of vitamin D and calcium, and either of each of them alone. We suggested effects of metabolism through induction of changes in the levels of various biomarkers that have influences in carcinogenesis including p53, heat shock proteins, iNOS and WT1 gene.
\end{abstract}

Keywords: Vitamin D, calcium, colon, colon cancer, p53, iNOS, WT1

\section{Introduction}

It has become common knowledge that an adequate supply of calcium and vitamin D is indispensable for optimal skeletal development as well as for maintenance of bone health (Peterlik et al., 2009).

However, it is of interest to note that inadequate intake and absorption of calcium as well as a compromised vitamin D status are not only well-known risk factors for skeletal disorders, but also contribute to the infectious, chronic inflammatory and autoimmune diseases (Peterlik and Cross, 2005).

Vitamin D receptors (VDRs) are present in a large variety of cell types including: colonic mucosal cells, and these cells are able to convert 25$(\mathrm{OH}) 3$ into the active form of vitamin D (1,25- $(\mathrm{OH}) 2 \mathrm{D} 3)$ ( Kallay et al., 2005). 
Vitamin D regulates colonic proliferation, differentiation, apoptosis and angiogenesis, and the colonic vitamin D system is regulated by several known natural factors; the most important once is nutritional calcium (Cross et al., 2009)

Cell death by apoptosis occurs as a part of a natural regulatory process in the body, serving to establish and maintain a proper control of the cellular turnover. Tumor suppressor act to maintain tissue homeostasis, that is, to control the apoptosis process and p53 protein is the most extensively studied tumor suppressor (Hansen et al., 2001; Grorge, 2011).

P53 gene has been found to be mutated in more than $50 \%$ of human cancers; and p53 protein is a very labile protein, with a short half-life, but it was recently demonstrated that WT1 protein play an important role to alter p53 stability (Asher et al.,2001).

The ability to maintain $\mathrm{O} 2$ homeostasis is essential to survival , so the intricately branched circulatory network of vascular endothelial and supporting cells is essential for transporting oxygen, nutrients and removal of carbon dioxide and metabolic products from cells, tissues and organs; formation of new blood vessels is divided into two components: vasculogenesis and angiogenesis (Hirota and Semenza, 2006).

Vascular epithelial growth factor (VEGF) signaling is required for the full execution of vasculogenesis and angiogenesis (Roskoski, 2007). Increasing age is associated with a reduced capacity to maintain homeostasis in all physiological systems and it might be that this results, in part at least, from a parallel and progressive decline in the ability to produce heat shock proteins (Pockley, 2001). Heat shock proteins play an important role in protein homeostasis, and they found in major cellular compartments (Tkacova, 2012). Directly or indirectly, 1, 25 dihydroxyvitamin D controls more than 200 genes, include genes responsible for the regulation of cellular proliferation, differentiation, apoptosis and angiogenesis (Holick, 2007), but the effect of vitamin D with and without calcium was studies only on various types of cancer, of these, colon, breast, prostate, renal and ovarian cancer (Park et al , 2007; Peterlik et al., 2009; Cui Y. and Rohan T., 2006).

Thus no report was made discussing the physiological effects of vitamin D and calcium on colon, and especially their effect on the WT1 and P53 level and explains clearly the stability effect of WT1 on p53, the current work will be interested in evaluating the physiological activity of vitamin D with and without calcium on colon.

\section{The colon}

The large intestine, or colon, extends from the ileocecal valve to the anus, forming the small intestine on three sides, chyme from the ileum passes into cecum, at the beginning of the large intestine. Waste material then pass 
in sequence through the ascending colon, transverse colon, descending colon, sigmoid colon, rectum and anal canal (Fox, 2011).

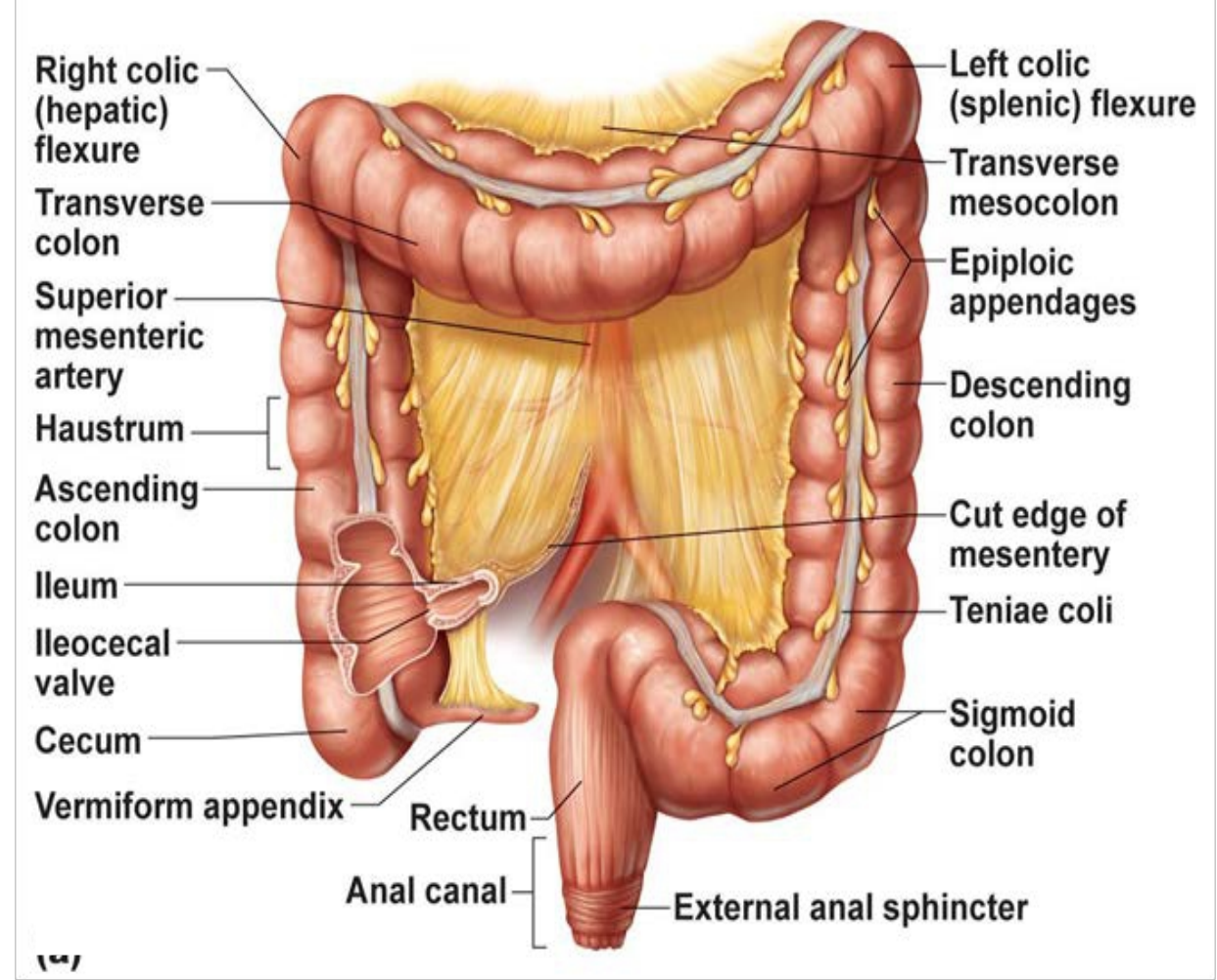

Figure 1. The large intestine. The different regions of the large intestine(colon)(Fox, 2011)

The colon, has little or no digestive function, but it does absorb water and electrolytes from remaining chime, as well as several B complex vitamins and vitamin $\mathrm{K}$ (Fox, 2011) The colonic mucosa cells are also able to convert 25-(OH)3 into the active form of vitamin D (1,25-(OH)2 D3) ( Kallay et al., 2005) whereas 25(OH)D3 serum concentration is a major determinant of 1,25-(OH)2 D3 synthesis in colonic mucosa, which expresses the vitamin D receptor and both the synthesizing (CYP27B1) and catabolic (CYP24A1) hydroxylases (Cross and Kallay, 2009).

\section{Calcium}

Calcium is an important mineral component of our diet. It is chiefly available to us in milk and milk products, certain seafood, legumes and some vegetables. The name of calcium is derived from Latin word calas, meaning lime, was known as early as the first centuries when the ancient Romans prepared lime as $\mathrm{CaO}$. It was not actually isolated until 1808 in England when Humphrey Davy electrolyzed a mixture of lime and mercuric oxide. Davy was trying to isolate calcium along with magnesium, strontium and 
barium. Out of alkaline earth group, $\mathrm{Ca}++$ has achieved greatest use and tonnage (Bhatia, 2008; Pravina et al., 2013).

The body contains about $2 \%$ of calcium and about $98 \%$ of this is in the bones which act as a reservoir of calcium for use throughout the body. Different levels of daily $\mathrm{Ca}++$ intake according to age and sex. Minimum of $1,000 \mathrm{mg}$ calcium per day is required for healthy adults until age 60 years, while higher values apply for people of advanced age or women during pregnancy and lactation as well as after menopause (Fox, 2009; Peterlik et al., 2009).

\section{Calcium forms}

Total Calcium in serum and extracellular fluid is divided into three forms: a) ionized form, which comprises approximately 50\% of the total; b) calcium bound with protein, which represent approximately $45 \%$ of the total, and c) calcium that is complexed to anions such as citrate, bicarbonate or phosphate, this form comprises approximately 5\% of the total (Sharan et al., 2008).

The protein- bound calcium does not diffuse out of the blood and it is bound principally to negatively- charged sites of albumin with smaller amount bound to globulins (Pravina et al., 2013).

\section{Calcium homeostasis}

The body has a fine tuned system to regulate the level of calcium in the extracellular fluid; this system is termed the calcium homeostatic system, it involves several hormones and organs. The hormones involved the parathyroid hormone, vitamin D and calcitonin. And the organs involved the parathyroid gland, kidney, skeleton and intestine (Tfelt, 2008).

\section{Biological activity of calcium}

Calcium is essential to maintaining total body health; the body needs it every day not just to keep bones and teeth strong, it is very important extracellularly as well as intracellularly. In the cell $\mathrm{Ca}++$ serves as an intracellular messenger that participates in muscle contraction, neurotransmission, and enzyme activity; in outside the cell, the extracellular calcium ion is important as the cation participates in blood clotting, contributes to maintaining the membrane potential across the cell membrane, neurotransmitter release, and maintenance skeletal integrity (Shara et al., 2008; Pravina, 2013).

\section{Vitamin D}

The term "vitamin D" was first used in 1922 when McCollum and colleagues reported the identification of a new lipid- soluble substance, 
which they named vitamin D. This finding appeared to be of great importance as the new vitamin showed to be capable of preventing rickets, a bone disease, which at that time had reach epidemic proportion (Hansen et al., 2001; Holick, 2006).

The identification of vitamin $\mathrm{D}$ led to intensive search for the active substrate of this vitamin, in 1931 Askew and colleagues succeeded in isolating vitamin D2 from irradiated plant sterols, later in 1936, vitamin D3 was identified by Windaus and colleagues, but it was not until the 1971where $(1,25(\mathrm{OH}) 2 \mathrm{D} 3)$ the metabolically active form of vitamin D3 was identified by Holick and colleagues (Holick, 2003; Kochupillai, 2008).

\section{Vitamin D forms}

There are two naturally occurring forms of vitamin $\mathrm{D}$ are cholecalciferol (vitamin $\mathrm{D}_{3}$ ) that is synthesized in the skin of animals and humans in response to sunlight. and ergocalciferol (vitamin $\mathrm{D}_{2}$ ) that is photochemically synthesized in plants (Cui and Rohan, 2006; Vuolo et al., 2012).

Recent studies in humans have provided evidence that vitamin $\mathrm{D}_{3}$ is more efficient than vitamin $\mathrm{D}_{2}$ in increasing serum concentration of vitamin $\mathrm{D}$ and maintaining those levels for a longer time; also its metabolites have superior affinity for vitamin D- binding proteins in plasma. (Armas et al., 2004).

1, 25(OH) 2D3 the metabolically active form of vitamin $\mathrm{D}$ is structurally related to the classic steroid hormones, in contrast to the classic steroid hormones, the B-ring of the ring structure has undergone fission by breakage of the 9,10 carbon bond and chain and is conformationally more flexible than the classic steroid hormones (Hansen et al., 2001).
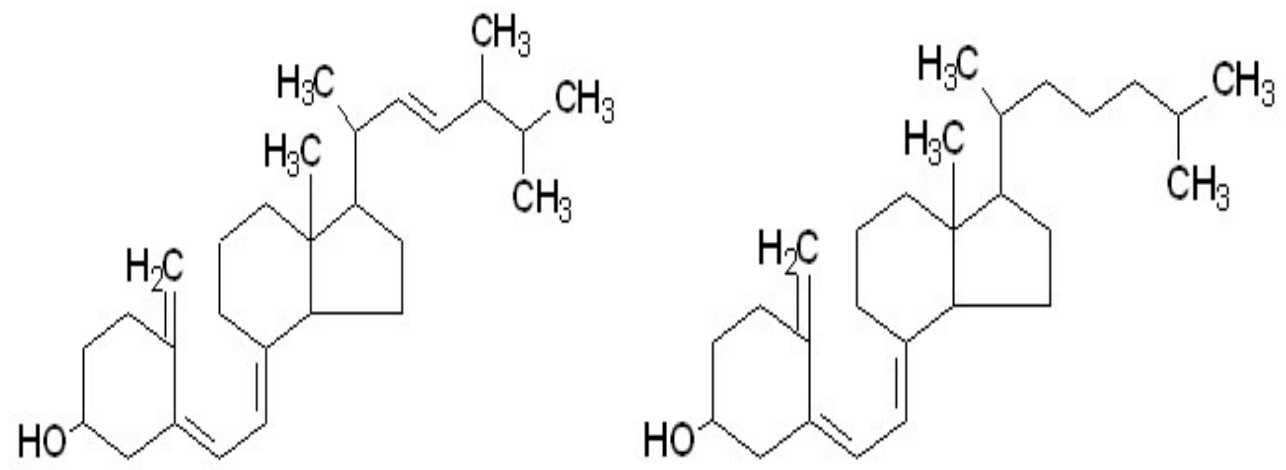

Vitamin D2

Vitamin D3

Figure 2: The chemical structure of vitamin D2 and vitamin D3 (Mercier and Luu, 2009). 


\section{Synthesis of Vitamin D}

Up to $80 \%$ of the body's need for vitamin D3 can be met by UV-Binduced synthesis of vitamin D3 in the skin (Cross et al., 2009).

The precursor of vitamin D3 is synthesized in the skin from 7dehydrocholesterol, this is an important molecule in the synthesis of cholesterol and is present mainly in the stratum spinosum and stratum basal of the epidermis; 7-dehydrocholesterol is a strong UV absorber with $3 \lambda$ max around $290-310 \mathrm{~nm}$, and under UV irradiation it is partly photolysis to create previtamin D3, which immediately converted to vitamin D3 in a heatdependent process (Mercier and Luu, 2009).

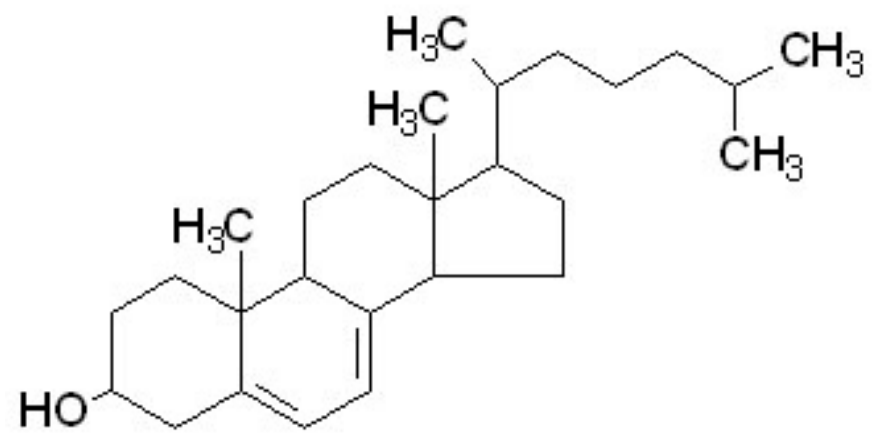

Figure 3: Chemical structure of 7-dehydrocholesterol (Mercier and Luu, 2009)

During prolong exposure to the sun; the synthesis of previtamin D3 reaches a plateau at concentration of about $10-15 \%$ of the original 7dehydrocholesterol concentration. In turn, previtamin D3 and vitamin D3 degrade into inactive photoproducts (Holick et al., 2011).

Vitamin D is poorly soluble in aqueous media and highly soluble in lipids, therefore it is transported in blood with the help of a binding carrier protein called vitamin D binding protein (DBP), plasma concentration of DBP is 20 times higher than the total amount of vitamin D metabolites.(Kochupillai, 2008)

After the synthesis of vitamin $\mathrm{D}$ in the skin from 7dehydrocholesterol or by intestine absorption of vitamin D2 from the dietary sources, the vitamin D is transported by DBP to adipose tissues for storage and for the liver where it is hydroxylated by the mitochondrial cytochrome P450 enzyme, 25-hydroxylase (CYP27A1), to 25- hydroxyvitamin D3 $(25(\mathrm{OH})$ D3) which is the major circulating form of vitamin D. The production of 25(OH) D3 is virtually uncontrolled and seems to correlate with substrate availability rather than with physiological needs. Therefore, measuring circulating $25(\mathrm{OH}) \mathrm{D} 3$ levels provides a useful indicator of the 
actual vitamin D status in the body and is widely applied in clinical practice (Hansen et al., 2001; Kallay et al., 2005; Lee et al., 2008).

Because 25(OH) D3 is biologically rather inactive, it has to be converted by $\mathrm{C} 1 \alpha$-hydroxylation to the hormonally active metabolite, 1,25 dihydroxyvitamin D3 (1, 25(OH)2 D3) also called Calcitriol. This occurs primarily in proximal tubule cells of the kidney catalyzed by another cytochrome P45 enzyme, 1 $\alpha$ - hydrolase (CYP27B1). (Holick, 2007; Cross et al., 2009). Although, other tissues and cell types have been shown to contain CYP27B1 and to produce $1,25(\mathrm{OH}) 2$ D3 from $25(\mathrm{OH}) \mathrm{D} 3$, the kidney is supposed to be the major site of production of circulating 1, 25(OH)2 D3, and in contrast to hepatic production of $25(\mathrm{OH}) \mathrm{D} 3$, renal synthesis of 1 , $25(\mathrm{OH}) 2 \mathrm{D} 3$ is a strictly regulated process that responds to the physiological needs for calcium and phosphate. Several agents have been suggested to be involved in this regulated, including protein kinase $\mathrm{C}$ (PKC), estrogens, prolactin, calcitonin and glucogorticoids, but the two most important physiological regulators are $1,25(\mathrm{OH}) 2$ D3 itself and parathyroid hormone(PTH) (Hansen et al ., 2001; Holick, 2006; Holick and chen, 2008)..

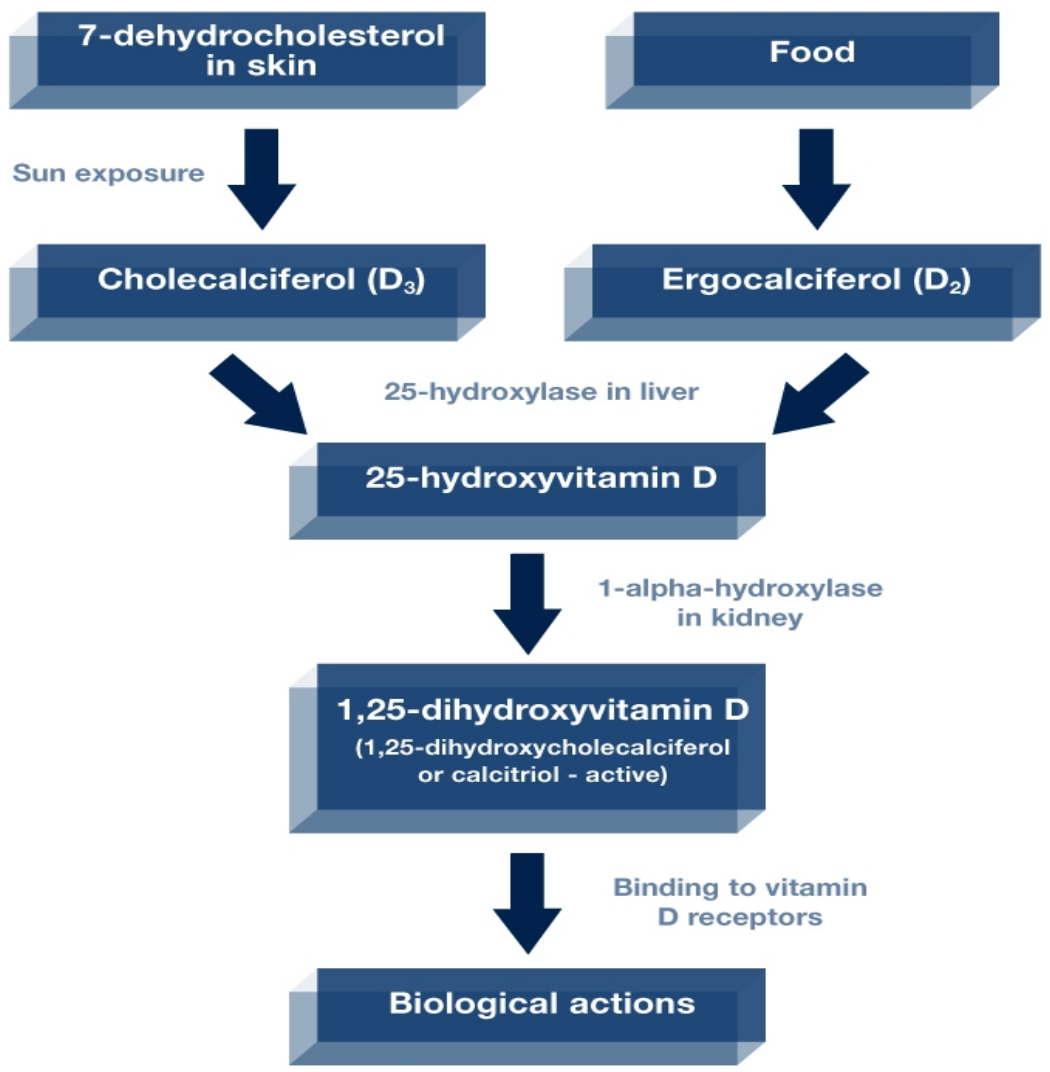

Figure 4: synthesis steps of 1, 25- dihydroxyvitamin D3 


\section{Biological activity of vitamin D}

The traditionally recognized role of vitamin $\mathrm{D}$ consist in the regulation of bone metabolism and calcium-phosphorus homeostasis, Besides these classic action, vitamin D3 has been shown to exert potent regulatory effects on growth and differentiation in a variety of cells which are not directly involved in the calcium metabolism ( Vuolo et al., 2012). An adequate supply of vitamin $\mathrm{D}$ is necessary to protect against several chronic diseases, such as rickets in children and osteoporosis in adult (Holick, 2006) hypertension, cardiovascular disease (Vacek et al., 2012). diabetes mellitus, allergic disorder, immunological disorder (Holick and Chen, 2008) and secondary hyperparathyroidism (Gonz et al., 2013).

\section{Role of vitamin $D$ in calcium homeostasis}

$1,25(\mathrm{OH}) 2 \mathrm{D} 3$ is the biologically active form of vitamin D responsible for maintaining

calcium and phosphorus homeostasis. It enhances the efficacy of small intestine to absorb calcium and phosphorus; it accomplishes this by interacting with its nuclear receptor, the vitamin $\mathrm{D}$ receptor (VDR) in the small intestinal cells (Fox, 2011).

The 1, 25(OH)2 D3 -VDR structure complexes with retinoic acid X receptor (RXR) in the nucleus. The1, 25(OH)2 D-VDR-RXR complex binds to the vitamin D-responsive element (VDRE) for the epithelial calcium channel. The increased expression of the calcium channel permits more calcium to enter the cell, where the vitamin D-dependent calcium-binding protein calbindin 9K helps calcium's translocation into the bloodstream (Holick, 2006; Kochupillai, 2008).

When dietary calcium is inadequate, vitamin $\mathrm{D}$ helps maintain calcium homeostasis by interacting with the VDR in osteoblasts to induce the expression of the plasma membrane protein receptor activator of NF- $\mathrm{B}$ ligand (RANKL). The RANK on the plasma membrane of preosteoclasts binds RANKL, which induces the preosteoclast to become a mature osteoclast; the mature osteoclast releases hydrochloric acid and collagenases to dissolve bone and release its precious calcium and phosphorus stores into the circulation (Holick, 2006; Holick, 2007). Apoptosis

Apoptosis is a type of physiological cell death that occurs during development normal tissue or as a result of different cellular insults (Hansen et al., 2001).

The term "apoptosis" was introduced by Kerr and his colleagues in 1971 to describe the morphological changes of this characteristic form of cell death; these changes involve chromatin condensation, cytoplasmatic and nuclear blabbing, eventually cellular demise without loss of membrane 
integrity and digestion of the resulting small vesicles by macrophages (Maximov and Maximov, 2008).

Apoptosis is triggered by a number of factors which act as through two major apoptotic pathways: extrinsic and intrinsic pathway; both pathways are activated by tumor-suppressor p53 protein (Fridman and Lowe, 2003).

These factors include some particular receptors on the cell surface (referred to as "death" receptors) and the components of extrinsic and intrinsic pathways, regulated by the members of a family of proteins called Bcl-2 (promoting mitochondria changes with release of cytochrome-c) Whichever pathway is induced, both lead to activation of the members of highly selective proteases referred to as "caspases". That is why the extrinsic pathway is otherwise known as the "death receptor pathway" and the intrinsic pathway -as the "mitochondrial pathway" (Bai and Zhu, 2006; Maximov and Maximov, 2008).

\section{The $\mathbf{p} 53$ protein}

Tumor suppressor p53 protein, also known as "TP53"(tumor protein) is a product of the tumor suppressor gene p53 located at the short arm of the human chromosome 17; the name is due to its molecular mass: it runs as a 53 kDa (kilo Dalton) protein (Maximov and Maximov, 2008).

The tumor suppressor p53 is a transcription factor involved in maintaining genomic integrity by controlling cell cycle and cell survival (Somasundaram and Deiry, 2000), p53 is a very labile protein, with a halflife of about 20 minutes, The rapid degradation of p53 is largely achieved through the ubiquitin proteasome pathway. the accumulation of p53 in response to DNA damage and other types of stress occurs mainly through posttranslational modification Proteins known to alter p53 stability include HPV16-E6. simian virus 40 (SV40) large $\mathrm{T}$ antigen adenovirus E1ByE4orf6, WT1, and Mdm2 .Whereas association of SV40 T antigen or WT1 with p53 increases p53 stability (Asher et al., 2001).

On cellular stress; particularly that induced by DNA damage, p53 can arrest cell cycle progression, thus, allowing the DNA to be repaired or it can lead to apoptosis (Fridman and Lowe, 2003).

These functions are achieved, in part, by the transactivation properties of $\mathrm{p} 53$, which activate a series of genes involved in cell cycle regulation, in cancer cells that bear a mutant $\mathrm{p} 53$, so this protein is no longer able to control cell proliferation, which results in inefficient DNA repair and the emergence of genetically unstable cells (Soussi, 2000). 


\section{Angiogenesis}

Angiogenesis is a complex process that involved the activation, proliferation, and directed migration of endothelial cells to form new capillaries from existing blood vessels (Oklu et al., 2010).

Angiogenesis is absolutely essential for embryonic growth, tissues growth and repair, an imbalance in this process contributes to numerous malignant, inflammatory, ischaemic, infectious and immune disorders (Carmeliet, 2005).

The rate of this process is increased during wound healing and tissue repair, during ovarian corpus luteum formation, and during placental development establishing pregnancy (Roskoski, 2007).

This process is regulated by both endogenous activators and inhibitors, and this regulate is under stringent control, there are about 30 known endogenous pro-angiogenic factors. The VEGF/FEGFR (vascular endothelial growth factor/VEGF receptor) family is the most studies regulators of vascular development (Plank and Sleeman, 2003).

\section{Vascular endothelial growth factor (VEGF)}

VEGF is an essential regulator of angiogenesis process; the three key activators of endothelial cell in angiogenesis are secretion of proteases, migration and proliferation. VEGF is capable of inducing all three of these (Oklu et al., 2010).

The VEGF family consists of six members: VEGF-A, VEGF-B, VEGF-C, VEGF-D, VEGF-E, and placental growth factor (PIGF). VEGF is so potent an angiogenesis activator that its expression must be precisely controlled both spatially and temporally for angiogenesis to proceed correctly (Plank and Sleeman, 2003).

There are three receptors protein-tyrosine kinase for VEGF family of ligands (VEGFR1, VEGFR2 and VEGFR3) and two non-enzymatic receptors (neuropilin-1 and -2) (Roskoski, 2007).

\section{Heat shock protein}

Heat shock proteins (HSPs) are a large class of proteins that have been conserved throughout evolution and exist by prokaryote and eukaryote organisms, they play an important role in protein homeostasis, they are synthesized as a response to a number of factors, for example, elevated temperature, UV radiation, bacterial and viral infection, heavy metal and others (Latchman, 2003; Tkacova and Angelovicova, 2012).

Many of these proteins are synthesized by normal unstressed cells with their synthesis being further enhanced upon exposure to stress, for example hsp90 is one of the most abundant proteins in unstressed cells (Pockley, 2001). 
HSPs are categories into several families that are named on the basis of their approximate molecular mass (the $70 \mathrm{kDa} H s p 70,60 \mathrm{kDa} H s p 70$ and $90 \mathrm{kDa}$ Hsp 90) under physiological conditions, some of these proteins function as molecular chaperones or proteases that have a number of intracellular functions(Latchman, 2003).

Chaperones regulate changes in the protein arrangement through membrane during transport, they regulate conformation- arrangement of protein at the slight damage, chaperones inhibit of change of proteins conformation at increased temperature to $42^{\circ} \mathrm{C}$ in the cell, both in normal and in stressed cells there will be a need to degrade proteins which have become abnormally folded and cannot be rescued by the action of chaperone proteins, it is therefore of interest that proteases such as the ubiquitindependent proteasome which plays a critical role in protein turnover by being linked to proteins marked for degradation (Latchman, 2003; Tkacova and Angelovicova, 2012).

\section{The Wilms'-Tumor suppressor protein (WT1)}

The Wilms'- tumor suppressor gene WT1 was originally identified as a tumour suppressor involved in the formation of Wilms' tumour of the kidney, and was additionally found to be associated with genitourinary disorders such as Denys Drash and Frasier syndromes ( Hartkamp and Roberts, 2008; Ohno et al., 2009).

The biology of WT1 is complex, since in addition to its function as a tumour suppressor; this gene has multiple roles during development and maintenance of body function (Wagner et al., 2003).

This gene encodes a zinc finger transcription factor (Wilms'- Tumor "WT1") that plays important roles in cell growth and differentiation .Although WT1 gene was categorized at first as a tumor-suppressor gene, it was recently demonstrated that the wild type WT1 gene has also emerged as an oncogenic factor influencing proliferation and apoptosis in a large variety of adult cancers (Hohenstein and Hastie, 2006; Ohno et al., 2009).

WT1 is expressed during mammalian embryonic development in many tissues, and the complexity of WT1 action during development is reflected on the molecular level. Post-transcriptional modifications of the Wt1 pre-mRNA lead to the production of up to 24 different isoforms, may result from a combination of alternative translation start sites, alternative RNA splicing and RNA editing, which seem to serve distinct but also overlapping cellular and developmental functions (Wagner et al., 2003; Hohenstein and Hastie, 2006).

It is now well established that WT1 can bind, via its zinc fingers, to the promoter regions of $>20$ downstream target genes, and the effects of WT1 on transcription can be either repressing or activating, depending on the 
cell type and the target gene with which WT1 interacts (Mrowka and Schedl, 2000).

\section{References:}

Armas L., Hollis B. and Heaney R. (2004). Vitamin D2 is much less effective than vitamin D3 in humans. The Journal of Clinical Endocrinology and Metabolism. 89(11): 5387-5391.

Asher G., Lotem J., Cohen B., Sachs L. and Shaul Y. (2001) Regulation of p53 stability and p53-dependent apoptosis by NADH quinone oxidoreductase 1. Proceeding of the National Academy of Sciences of The United States of America. 98(3): 1183-1193.

Ansary A., Palacios C., De-Regil L. and Rosas J. (2010) Vitamin D supplementation for women during pregnancy. John Wiley \& Sons, Ltd. 6: 25-58.

Carmeliet P. (2005) Angiogenesis in life, disease and medicine. Nature. 438: 932-936.

Cui Y. and Rohan T.(2006) Vitamin D, Calcium, and Breast Cancer Risk: A Review. Cancer Epidemiology Biomarkers and Prevention. 15(8): 14271437.

Cross H., Nittke T. and Peterlink M. (2009). Modulation of vitamin D synthesis and catabolism in colorectal mucosa: Anew target for cancer preventation. Anticancer Research. 29: 3705-3712.

Cross H. and Kallay E. (2009). Regulation of the colonic vitamin D system for prevention of tumor progression. Future Oncology.5(4): 493-507.

Fridman J. and Lowe S. (2003) Control of apoptosis by p53. Oncogene. 22: 9030-9040.

Fox S. (2011). Human physiology.2ed.McGraw-Hill. pp: 685-688.

Gonz L., Rodr A., Estaire P., Ruiz C. and Ortega R. (2013). Vitamin D intake and dietary sources in a presentative sample of Spanish adults. Journal of Human Nutrition and Dietetics. 26(1): 64-72.

Grorge P. (2011). P53 How crucial is its role in cancer? International Journal of Current Pharmaceutical Research. 3(2): 19-25.

Hansen C., Binderup L., Hamberg K. and Carlberg C. (2001). Vitamin D and cancer: effect of 1, 25(OH)2 D3 and its analogs on growth control and tumorigenesis. Frontiers in bioscience. 6: 820-848.

Holick M. (2003). Vitamin D: A Millenium Perspective. Journal of Cellular Biochemistry 88:296-307.

Holick M. (2006). Resurrection of vitamin D deficiency and rickets. The Journal of Clinical Investigation.116(8): 2062-2072.

Hohenstein P. and Hastie N.(2006). The many facets of the Wilms' tumor gene, WT1. Human molecular genetics. 15(2): 196-201. 
Hirota K. and Semenza G. (2006). Regulation of angiogenesis by hypoxiainducible Factor 1. Critical Reviews in Oncology/Hematology.59: 15-26. Holick M. (2007). Vitamin D deficiency. The New England Journal of Medicine. 81: 266- 357.

Holick M. and Chen T. (2008). Vitamin D deficiency: a worldwide problem with health Consequences. American Society for Nutrition.87:1080-1086.

Hartkamp J. and Roberts G.(2008). The role of the Wilms' tumor-suppressor protein WT1 in apoptosis. Biochemical Society Transactions. 36: 629-631.

Kochupillai N. (2008). The physiology of vitamin D:Current concepts. Indian journal of Medical Research. 127: 256-262.

Kallay E., Bises G., Bajna E., Bieglmayer C., Gerdenitsch W., Steffan I., Kato S., Armbercht H. and Cross H.(2005). Colon- specific regulation of vitamin D hydroxylases- a possible approach for tumor prevention. Carcinogenesis, 26(9): 1581-1589.

Lee J., Keefe J., Bell D., Hensrud D. and Holick M.(2008). Vitamin D deficiency. Journal of The American College of Cardiology, 52(24): 19491956.

Latchman D (2003). Protective effect of heat shock proteins: potential for gene therapy. Gene Therapy and Molecular Biology.7: 245-254.

Mrowka C. and Schedl A.(2000). Wilms’ Tumor suppressor gene WT1: from structure to renal pathophysiologic features. Journal of the American Society of Nephrology.11: 106-115.

Mercier M. and Luu M.(2009). Metabolism of vitamin D in skin: Benefits for skin care Application. Journal of Cosmetics and Toiletries.124(10): 4046.

Maximov G. and Maximov K.(2008). The role of p53 tumor-suppressor protein in apoptosis and cancerogenesis. Biotechnology \& Biotechnological Equipment.22(2): 664-668.

Ohno S., Dohi S., Ohno Y., Kyo S., Sugiyama H., Suzuki N. and Inoue M.(2009). Immunohistochemical detection of WT1 protein in endometrial cancer. Anticancer Research. 29: 1691-1696.

Oklu R., Walker T., Wicky S. and Hesketh R. (2010). Angiogenesis and current anti- angiogenic strategies for the treatment of cancer. Journal of Vascular and Interventional Radiology. 21: 1791-1805.

Pockley A.(2001). Heat shock proteins in health and disease: therapeutic targets or Therapeutic agents? Experimental Reviews in Molecular Medicine. 3(23):1-21.

Plank M. and Sleeman B.(2003). Tumor-Induced angiogenesis. Journal of Theoretical Medicine.5: 137-153.

Peterlik M. and Cross H.(2005). Vitamin D and calcium deficits predispose for multiple chronic diseases. European Journal of Clinical Investigation. 35: 290-304. 
Park S. , Murphy S., Wilkens L., Stram D., Henderson B., and Kolonel L.(2007). Calcium, Vitamin D, and Dairy Product Intake and Prostate Cancer Risk. American Journal of Epidemiology. 166(11): 1259-1269.

Roskoski R.(2007). Vascular endothelial growth factor (VEGF) signaling in tumor Progression. Oncology Hematology.62:179-213.

Peterlik M., Grant W. and Cross H.(2009). Calcium, vitamin D and Cancer. Anticancer Research. 29: 3687-3698.

Pravina P., Sayaii D. and Avinash M.(2013). Calcium and its role in human body. International Journal of Research in Pharmaceutical and Biomedical Sciences, 4(2): 659-668.

Soussi T. (2000). P53 antibodies in the sera of patients with various types of cancer. Cancer Research.60: 1777-1788.

Sharan K., Siddiqui J., Swarnkar G. and Chattopadhyay N.(2008) Role of calcium- Sensing receptor in bone biology. Indian Journal of Medical Research, 127: 274-286.

Tfelt J.(2008). The role of calcium-sensing receptor and signaling pathway in the Pathophysiology in two in vitro models of malignant hypocalcaemia. Danish Medical Journal. 55(1): 17-35.

Tkacova J. and Angelovicova M.(2012). Heat shock proteins(HSPs) Review. Animal Science and Biotechnology.45(1): 349-353.

Vuolo L., Somma C., Faggiano A. and Colao A.(2012). Vitamin D and Cancer. Frontiers in Endocrinology. 3: 58-70.

Wagner K., Wagner N. and Schedl A.(2003). The complex life of WT1. Journal of Cell Science.116: 1653-1658. 